\title{
Multi-condition M/EEG inverse modeling with sparsity assumptions: how to estimate what is common and what is specific in multiple experimental conditions
}

\author{
A. Gramfort ${ }^{1,2}$ \\ ${ }^{1}$ INRIA, Parietal Project Team, Saclay, France \\ ${ }^{2}$ INRIA, Odyssee Project Team, Sophia-Antipolis, France
}

\begin{abstract}
M/EEG inverse modeling with distributed dipolar source models and penalizations with sparsity inducing norms (e.g. $\ell_{1}$ with MCE [1], $\ell_{0}$ with FOCUSS [2], $\ell_{2}-\ell_{1}$ [3]) offer a way to select a set of active dipoles. Indeed, sparsity inducing norms lead to solutions where most of the sources are set to zero and the remaining non zero sources form the set of estimated active dipoles. When running cognitive studies multiple experimental conditions are usually involved and cognitive hypothesis classically consist in quantifying the difference between these conditions. The problem is that when a sparse inverse solver is used independently for each experimental condition, it happens that the selection of dipolar sources is not consistent across conditions, thus limiting further analysis. Even if all conditions share a common dipolar source, due to noise, it can happen that such solvers do not select exactly the same dipole but two neighboring ones. To circumvent this limitation, we propose in this contribution to run the inverse computation with all the experimental conditions simultaneously. We use a penalization that achieves a joint selection of active dipoles while estimating two parts in the reconstructed current distributions: a part that is common to all the different conditions and a part that is specific to each condition. The penalization used in the inverse problem is based on groups of $\ell_{2}-\ell_{1}$ norms. The optimization is achieved with iterative least squares (iterative $\ell_{2}$ Minimum Norm) making the solver tractable on large datasets. The method is illustrated on toy data and validated on synthetic MEG data reproducing activations appearing for somesthesic finger stimulations. We call our solver SMC (Sparse Multi-Condition).
\end{abstract}

Keywords- MEG, EEG, Inverse Problem, Sparse prior IRLS

\section{INTRODUCTION}

Distributed source models in Magnetoencephalography and Electroencephalography (collectively M/EEG) use the individual anatomy derived from high resolution anatomical Magnetic Resonance Images (MRI) [4]. They consist in sampling the automatically segmented cortical ribbon with a high number of equivalent current dipoles (ECD). Each dipole adds linearly its contribution to the measured signal leading to a linear solution to the forward problem. The measurements $M \in \mathbb{R}^{d_{m} \times d_{t}}$ ( $d_{m}$ number of sensors and $d_{t}$ number of time instants) are obtained by multiplying the current source amplitudes $X \in \mathbb{R}^{d_{x} \times d_{t}}$ ( $d_{x}$ number of dipoles) by a forward operator $G \in \mathbb{R}^{d_{m} \times d_{x}}$, called the lead field matrix, i.e., $M=G X$.

Solving the forward problem consists in computing $G$ taking into account the electromagnetic properties of the head [5], while solving the inverse problem consists in estimating the neural currents $\hat{X}$ that explain the observed measurements. However, this latter problem is strongly ill-posed. It implies that $\hat{X}$ can only be computed if priors are set on the solution. Standard priors assume that a weighted $\ell_{2}$ norm of $\hat{X}$, denoted $\|\hat{X}\|_{\mathbf{w} ; F}$ (Frobenius norm), is small. The estimated distribution of cortical currents $\hat{X}$ is obtained by solving:

$$
\hat{X}=\underset{X}{\arg \min }\|M-G X\|_{F}^{2}+\lambda \phi(X), \lambda \in \mathbb{R}_{+}
$$

with $\phi(X)=\|X\|_{\mathbf{w} ; F}^{2}$ and $\|X\|_{\mathbf{w} ; F}^{2}=\sum_{t=1}^{d_{t}} \sum_{i=1}^{d_{x}} w_{i} x_{i t}^{2}$, $\mathbf{w}=$ $\left(w_{i}\right)_{i} \in \mathbb{R}_{+, *}^{d_{x}}$. Such priors provide the grounds of what are called in the M/EEG literature Minimum Norm (MN) inverse solvers [6, 5] and the noise normalized variants, e.g., dSPM [7]. Such standard solvers are fast to compute since $\ell_{2}$ priors lead to linear inversion. Indeed $\hat{X}$ is obtained by multiplication $M$ with a matrix $H_{\mathrm{w}}$ :

$$
\hat{X}=W^{-1} G^{T}\left(G W^{-1} G^{T}+\lambda I d\right)^{-1} M=H_{\mathbf{w}} M,
$$

where $I d$ stands for the identity matrix and $W$ is a diagonal matrix whose diagonal elements are the weights $w_{i}$. However, there are shortcomings of such simple solvers. The main critic is that they tend to smear the estimated current distributions over widely extended cortical regions, limiting the spatial resolution of the reconstructed sources. To circumvent this limitation and obtain more focal activations, alternative strategies based on general $\ell_{p}$ norms have been proposed. With $\ell_{p}$ norms, a value of $p \leq 1$ induces "sparsity", i.e., , a small number of sources with non-zero amplitudes, while with $\ell_{2}$ all sources have non-zero amplitudes. This observation led to the development of FOCUSS [2] and MCE [1] that 
work instant by instant, and more recently to a promising spatiotemporal inverse method based on a mixed $\ell_{2}-\ell_{1}$ norm [3]. We call such solvers sparse solvers and the set of estimated active dipoles the active set.

During an experiment, a subject is generally asked to perform different cognitive tasks or to respond to various external stimulations. They are referred to as different experimental conditions. Neuroscience questions ofter require to quantify the difference between these conditions, i.e., to assess what are the different and the common active brain regions involved in the different conditions. Let us denote by $M^{k}$ the measurements for condition $k$ and $d_{k}$ the number of conditions. With linear inverse solvers, a contrast map between condition $k$ and condition $l$ can be obtained simply by computing: $\hat{X}^{k}-\hat{X}^{l}=H M^{k}-H M^{l}=H\left(M^{k}-M^{l}\right)$.

With a sparse solver that provides better spatial resolution, the contrast $\hat{X}^{k}-\hat{X}^{l}$ can also be computed but it is likely to fail. Because of noise, the active set for condition $k$ is likely to be different than the active set for condition $l$ even if both conditions share a common active region. The aim of the solver detailed in this contribution is to circumvent this limitation. To do so, we propose a solver based on $\ell_{2}-\ell_{1}$ mixed norms that inverts all the conditions simultaneously as in [8] for the problem of functional mapping with M/EEG. By doing so, we want to estimate what are the common and specific active regions in all conditions.

The rest of this contribution consists of two parts. Section [I, introduces the solver and briefly sketches the implementation details. Section ПI, presents some simulation results on two datasets: toy data and synthetic MEG data reproducing activations appearing for somesthesic stimulations of different fingers. Quantitative validation is provided on the realistic dataset.

\section{METHOD}

In [3], the problem solved corresponds to (1) where $\phi(X)$ equals to $\|X\|_{21}$. This $\ell_{21}$ mixed norm is defined for a matrix $X \in \mathbb{R}^{d_{x} \times d_{t}}$ by $\|X\|_{21}=\sum_{i=1}^{d_{x}}\left\|X_{i}\right\|_{2}=\sum_{i=1}^{d_{x}} \sqrt{\sum_{t=1}^{d_{t}} x_{i t}^{2}}$.

When penalizing the inverse problem with the $\ell_{21}$ norm the solution $\hat{X}_{21}$ has a sparse structure where only a few rows have non-zero coefficients. These rows form the active set $\mathscr{A}\left(\hat{X}_{21}\right)$ of the $\ell_{21}$ solution: $\mathscr{A}\left(\hat{X}_{21}\right)=\left\{i\right.$ s.t. $\hat{x}_{i t} \neq$ 0 for all $t\}$. The reason for this sparse structure is that the $\ell_{21}$ norm groups row coefficients in different $\ell_{2}$ norms: $\left\|X_{i} \cdot\right\|_{2}$.

When considering different experimental conditions, indexed by $k$, the $\ell_{21}$ solver provides the solutions $\hat{X}_{21}^{k}$ whose active sets are $\mathscr{A}\left(\hat{X}_{21}^{k}\right)$. Due to noise, is it likely that the two estimated active sets $\mathscr{A}\left(\hat{X}_{21}^{k}\right)$ and $\mathscr{A}\left(\hat{X}_{21}^{l}\right)$ are not exactly equal, even if the generators in both conditions are exactly the same. One way to go around this issue consists in computing the inverse problem on all conditions simultaneously by solving the problem:

$$
\hat{X}=\underset{X}{\arg \min } \frac{1}{2}\left\|\sum_{k=1}^{d_{k}} M^{k}-G X^{k}\right\|_{F}^{2}+\lambda\|\underline{X}\|_{21}, \lambda \in \mathbb{R}_{+}
$$

where the matrix $\underline{X} \in \mathbb{R}^{d_{x} \times d_{k} d_{t}}$ is obtained by concatenating horizontally all the $X^{k}$. By doing so, one forces $\mathscr{A}\left(\hat{X}_{21}^{k}\right)$ to be the same for all $k$.

In practice, this latter constraint is too strong as different conditions necessarily have specific current generators in addition to the common ones. This observation suggests that one could estimate each $\hat{X}^{k}$ in two components: a component $\hat{X}_{c}^{k}$ whose active set is common between all conditions and a component $\hat{X}_{s}^{k}$ that is specific. The problem addressed becomes:

$$
\begin{aligned}
\left(\hat{X}_{c}^{k}, \hat{X}_{s}^{k}\right)_{k} & =\underset{\left(X_{c}^{k}, X_{s}^{k}\right)_{k}}{\arg \min } \frac{1}{2}\left\|\sum_{k=1}^{d_{k}} M^{k}-G X_{c}^{k}-G X_{s}^{k}\right\|_{F}^{2} \\
& +\lambda \rho\left\|\underline{X}_{c}\right\|_{21}+\lambda(1-\rho) \sum_{k=1}^{d_{k}}\left\|X_{s}^{k}\right\|_{21}
\end{aligned}
$$

where $\lambda \in \mathbb{R}_{+}$and $\rho \in[0,1]$. Once $\left(\hat{X}_{c}^{k}, \hat{X}_{s}^{k}\right)_{k}$ are estimated one can get access to the components of the measurements that are common and specific, $\left(\hat{M}_{c}^{k}, \hat{M}_{s}^{k}\right)_{k}$, defined by $\hat{M}_{c}^{k}=$ $G \hat{X}_{c}^{k}$ and $\hat{M}_{s}^{k}=G \hat{X}_{s}^{k}$.

Implementation We solve the optimization problem in (4) using iterative Weighted Minimum Norm computations (2) where the weights are updated at each iteration. The approach is similar to the FOCUSS solver and is usually referred to as IRLS (Iterative Reweighted Least Squares). Some details can be found in section 4.2.1 in [9]. Once the active set is estimated, the time series are obtained by running an ordinary least square constrained to the active set, which is no longer an ill-posed problem as the active set size is smaller than the number of sensors.

\section{Simulation StUdY}

To illustrate the method, we designed a toy dataset with 3 conditions, 1 common generator and 1 specific generator in each condition. The time series for each source is simulated to be smooth and last for $100 \mathrm{~ms}$ with positive and negative deflections similar to standard evoked responses measured by M/EEG. The toy dataset has $d_{m}=10$ sensors and 
$d_{x}=50$ possible sources. The matrix $G$ is random with normalized columns. The noiseless time series, used later as ground truth, $\left(\check{M}_{c}^{k}, \check{M}_{s}^{k}\right)_{k}$ with $\check{M}^{k}=\check{M}_{c}^{k}+\check{M}_{s}^{k}$ are presented in figure 1. Since activations are not necessarily time locked with the same delay across conditions, activation time series are perturbed with random time jitters. This is illustrated in figure 2 where the source time series are plotted. The matrices $X_{c}^{k}$ and $X_{s}^{k}$ can be visualized as images like in figure 3(a) or by looking at the active sets like in figure 3(b). One can observe the row structure of the sparsity pattern. Starting from $\left(\check{M}_{c}^{k}, \check{M}_{s}^{k}\right)_{k}$ to which has been added a Gaussian white noise $(\mathrm{SNR}=3)$, the sources $\left(\hat{X}_{c}^{k}, \hat{X}_{s}^{k}\right)_{k}$ are estimated taking as parameters $\lambda=0.01$ and $\rho=0.4$. It can be observed that we recover the true sparsity pattern, i.e., the good active sources. The associated estimated time series presented in figure 2(b) match with the ground truth presented in figure 2(a) The parameters were set empirically to maximize the fit with the ground truth. However, an optimal value for $\rho=1 /\left(1+\sqrt{d_{k}}\right) \approx 0.4$ can be analytically derived in the case where the time series of all generators have the same $\ell_{2}$ norm. If this does not hold, the parameter $\rho$ should be adapted.
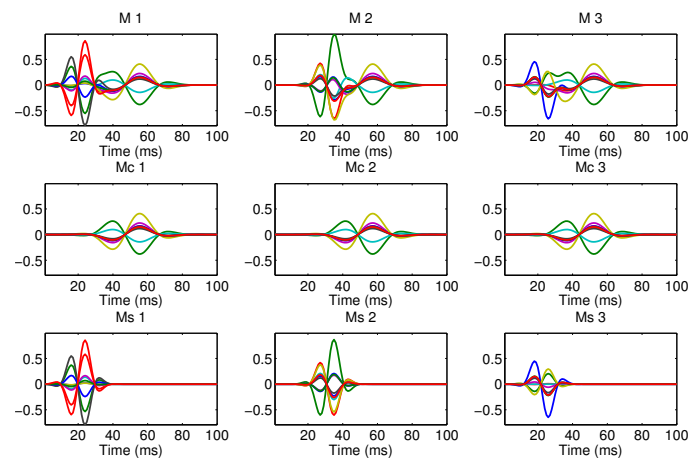

Figure 1: Noiseless toy dataset with 3 conditions, 1 common generator and

1 specific generator in each condition. Each column corresponds to a condition. The 1st row corresponds to $\check{M}^{k}$, the 2 nd to $\check{M}_{c}^{k}$ and the 3 rd to $\check{M}_{s}^{k}$.

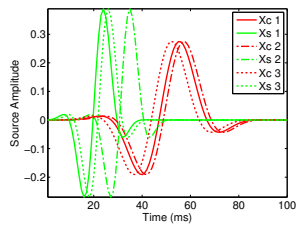

(a) Ground truth

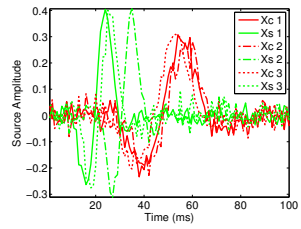

(b) Estimated
Figure 2: Source time series. In green are the time series for the specific sources and in red the time series for the common generator. (a) Series used for simulation before corrupting the signal with an additive Gaussian noise

(b) Estimated time series ( $\mathrm{SNR}=3, \lambda=0.01$ and $\rho=0.4$ ).

In order to quantitatively assess the performance of the method, we have conducted a simulation study with a realistic configuration. The matrix $G$ is obtained by solving an MEG
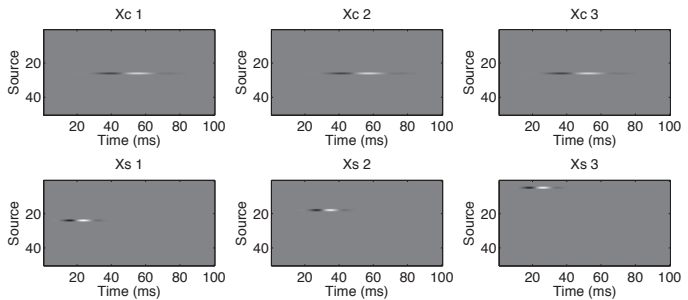

(a) Ground truth
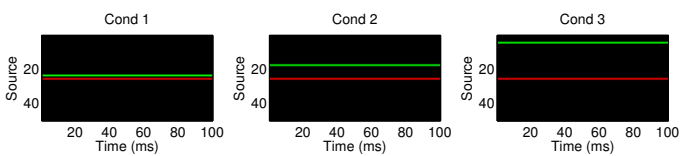

(b) Active Sets for all conditions

Figure 3: (a) Source amplitudes used for the toy data in all 3 conditions (b) Illustration of the active sets. In red is the active set common to all the $X_{c}^{k}$ and in green is the active set specific to the different $X_{s}^{k}$.
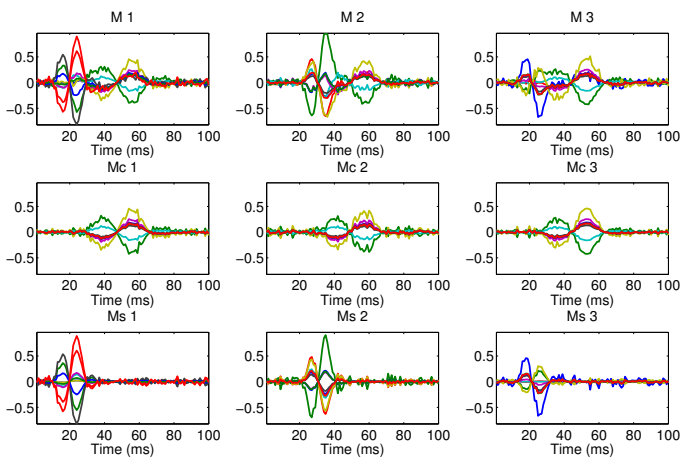

Figure 4: Estimated times series $\left(\hat{M}_{c}^{k}, \hat{M}_{s}^{k}\right)_{k}$. Each column corresponds to a condition. The 1 st row corresponds to $\hat{M}^{k}$, the 2 nd to $\hat{M}_{c}^{k}$ and the 3 rd to $\hat{M}_{s}^{k}$. One can observe a good match with the ground truth presented in figure 1

forward problem with a spherical headmodel. The sources are sampled over a cortically constrained domain consisting of a triangulated mesh of the cortical ribbon. In order to schematically reproduce activations appearing for somesthesic stimulations of two different right hand fingers, two specific generators have been positioned in the left primary somatosensory cortex (in green in figure 5) and one common generator has been positioned in the left parietal cortex. The time series of activations of these different generators are presented in figure 6(a) The computation has been carried out 10 times for multiple SNR values. One result of estimated source times series with SNR equals 1 is presented in figure 6(b) For each estimation, we chose to quantify the error using $\left(\hat{M}_{c}^{k}, \hat{M}_{s}^{k}\right)_{k}$ and the ground truth $\left(\check{M}_{c}^{k}, \check{M}_{s}^{k}\right)_{k}$. The errors $E_{c}$ and $E_{s}$ are defined by $E_{a}=\left\|\underline{\hat{M}}_{a}-\underline{\breve{M}}_{a}\right\|_{F} /\left(\left\|\underline{\hat{M}}_{a}\right\|_{F}+\left\|\underline{\breve{M}}_{a}\right\|_{F}\right) \in[0,1]$ where $\hat{M}_{a}\left(\right.$ resp. $\check{M}_{a}$ ) is obtained by concatenating horizontally the $\hat{M}_{a}^{k}\left(\right.$ resp. $\left.\breve{M}_{a}^{k}\right)$ and " $a$ " is set to " $c$ " or " $s "$.

The results of the simulations are presented in figure 7 One can observe that the method provides unbiased results 
at very high SNR. The errors obtained with SNR equal to 20 are almost 0 . As the SNR drops down, the errors increase but stay small until the SNR gets negative, which is acceptable.

In order to demonstrate that the portion of the active set that is common between conditions is more consistently recovered with the SMC solver, we have compared our results with the single condition spatiotemporal sparse solver proposed in [3]. We ran this solver on each condition successively and defined the common active sets as the intersection between all the $\mathscr{A}\left(\hat{X}_{21}^{k}\right)$. The $d_{k}$ specific portions of the active set for the $\ell_{21}$ solver were obtained by subtracting from each $\mathscr{A}\left(\hat{X}_{21}^{k}\right)$ the common portion. For both methods, the estimation error was quantified as the number of differences between the active sets obtained and the ground truth. Results are presented in figure 8 One can observe that the estimation of $\mathscr{A}_{c}$ is improved thanks to the SMC solver, especially at low SNR. This was the principal motivation for the development of this solver.

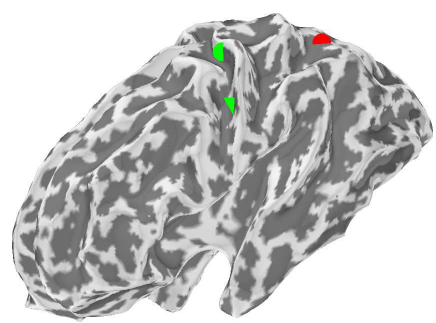

Figure 5: Synthetic source configuration with 2 specific generators in the primary somatosensory cortex (green sphere end green pyramid) and 1 common generator in the parietal cortex (red sphere).

\section{CONCLUSION}

In this contribution, we explained how sparse solvers can be used in M/EEG to go beyond simple localization by providing a way to estimate what are the specific and what are the common current generators involved in different cognitive tasks. By doing so we provide a principled and robust way to compare different conditions when using non-linear sparse inverse solvers.

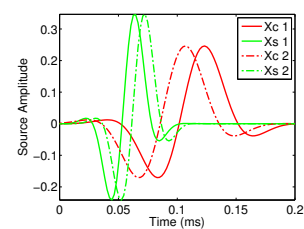

(a) Ground truth

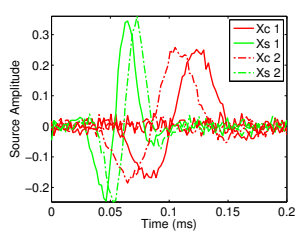

(b) Estimated
Figure 6: Source time series. In green are the time series for the specific sources and in red the time series for the common generator. (a) Series used for simulation before corrupting the signal with an additive Gaussian noise (b) Estimated time series $(\mathrm{SNR}=1, \lambda=5$ and $\rho=0.4)$.

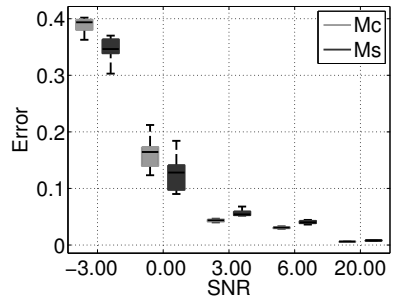

Figure 7: Estimation errors for different values of SNR (see text). Errors are provided for 10 repetitions of the experiment. The method has no estimation bias for high SNRs and presents low errors even at low SNRs.

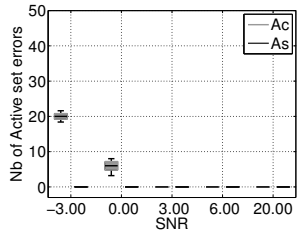

(a) $\mathrm{SMC}$

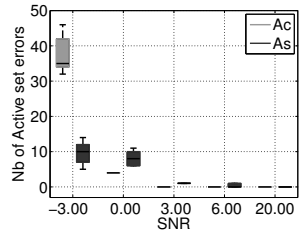

(b) Single condition $\ell_{21}$
Figure 8: Number of errors in the estimated active sets for both solvers: the $\ell_{21}$ solver [3] and the SMC solver. Results are presented for 10 repetitions of the experiment. It can be observed that the estimation of $\mathscr{A}_{c}$ is improved by the SMC solver especially at low SNR.

\section{REFERENCES}

1. Matsuura K., Okabe Y.. Selective minimum-norm solution of the biomagnetic inverse problem. IEEE Trans Biomed Eng. 1995;42:608-615.

2. Gorodnitsky I, George J, Rao B. Neuromagnetic source imaging with FOCUSS: a recursive weighted minimum norm algorithm Electroencephalography and clinical Neurophysiology. 1995.

3. Ou W, Hämaläinen M, Golland P. A Distributed Spatio-Temporal EEG/MEG Inverse Solver Neuroimage. 2009;44:932-946.

4. Dale A, Sereno M. Improved Localization of Cortical Activity By Combining EEG and MEG with MRI Cortical Surface Reconstruction Journal of Cognitive Neuroscience. 1993.

5. Hämäläinen M, Ilmoniemi R. Interpreting magnetic fields of the brain: minimum norm estimates Medical and Biological Engineering and Computing. 1994;32:35-42.

6. Wang J.-Z., Williamson S.J., Kaufman L.. Magnetic source images determined by a lead-field analysis: the unique minimum-norm leastsquares estimation Biomedical Engineering, IEEE Transactions on. 1992;39:665-675.

7. Dale A, Liu A, Fischl B, Buckner R. Dynamic statistical parametric neurotechnique mapping: combining fMRI and MEG for high-resolution imaging of cortical activity Neuron. 2000;26:55-67.

8. Gramfort A, Kowalski M. Improving M/EEG source localization with an inter-condition sparse prior in Proceedings ISBI 2009.

9. Gramfort A. Mapping, timing and tracking cortical activations with MEG and EEG: Methods and application to human vision. $\mathrm{PhD}$ thesis 2009 .

Author: Alexandre Gramfort Institute: INRIA NeuroSpin CEA

Street: Bat. 145, PC 156

City: Gif-sur-Yvette

Country: France

Email: alexandre.gramfort@inria.fr 